\title{
Amorphous Tin Oxide Nanohelix Structures Based Electrode for Highly Reversible Na-Ion Batteries
}

Il Yong Choi, ${ }^{\dagger, \S}$ Changshin Jo, ${ }^{\ddagger, \|, \S}$ Won-Gwang Lim,,${ }^{\ddagger, \perp}$ Jong-Chan Han, ${ }^{\dagger}$ Byeong-Gyu Chae, ${ }^{\dagger}$ Chan Gyung Park, ${ }^{\dagger}$ Jinwoo Lee, ${ }^{*, \perp}$ and Jong Kyu Kim ${ }^{*}, \dagger$

${ }^{\dagger}$ Department of Materials Science and Engineering, Pohang University of Science and Technology (POSTECH), Pohang 37673, Republic of Korea

${ }^{\ddagger}$ Department of Chemical Engineering, Pohang University of Science and Technology (POSTECH), Pohang 37673, Republic of Korea

${ }^{\perp}$ Department of Chemical and Biomolecular Engineering, Korea Advanced Institute of Science and Technology (KAIST), Daejeon 34141, Republic of Korea 


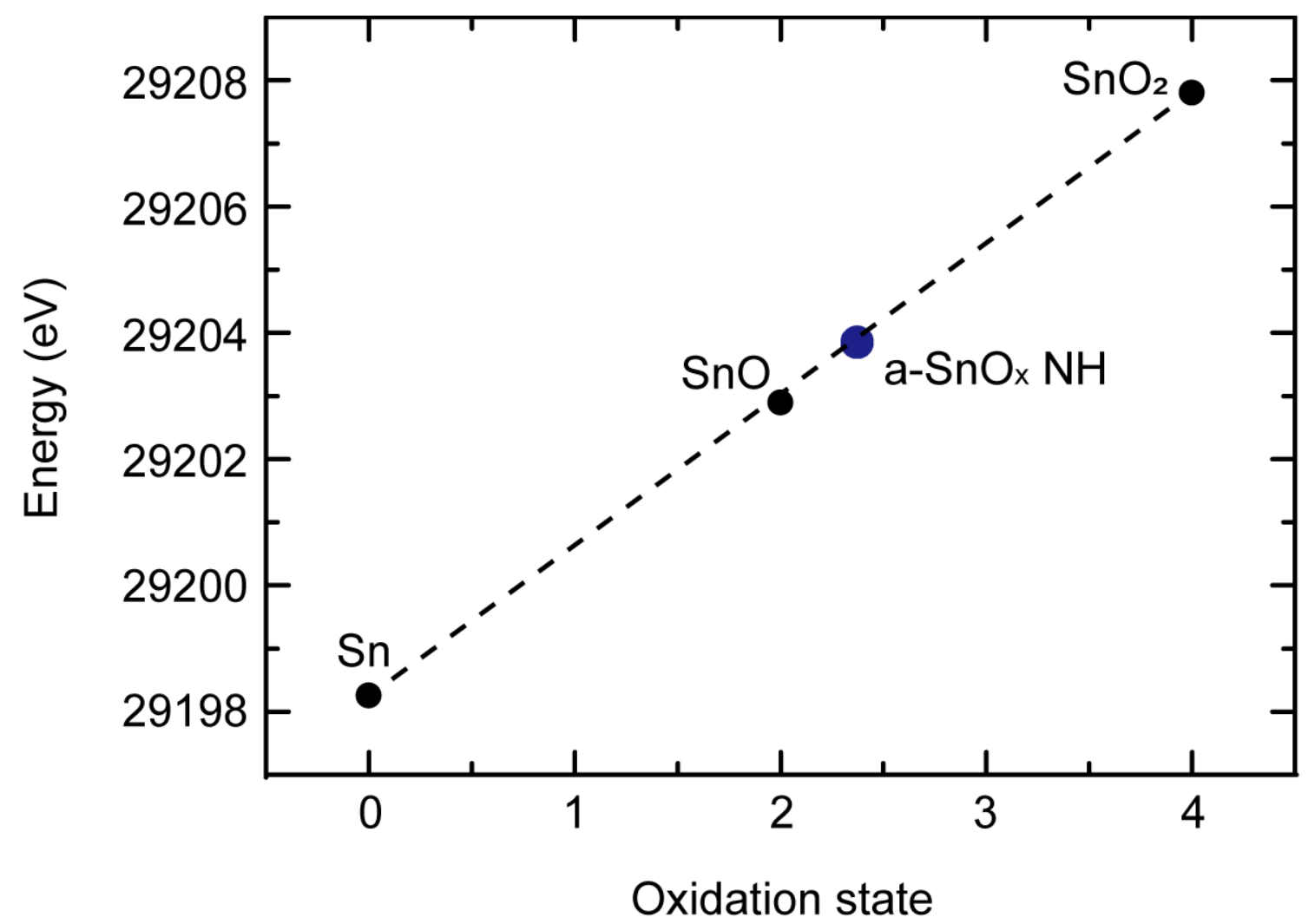

Figure S1. The average oxidation number of $\mathrm{Sn}$ in the a-SnOx $\mathrm{NH}$ electrode estimated from the XANES profiles in Figure 3b. 

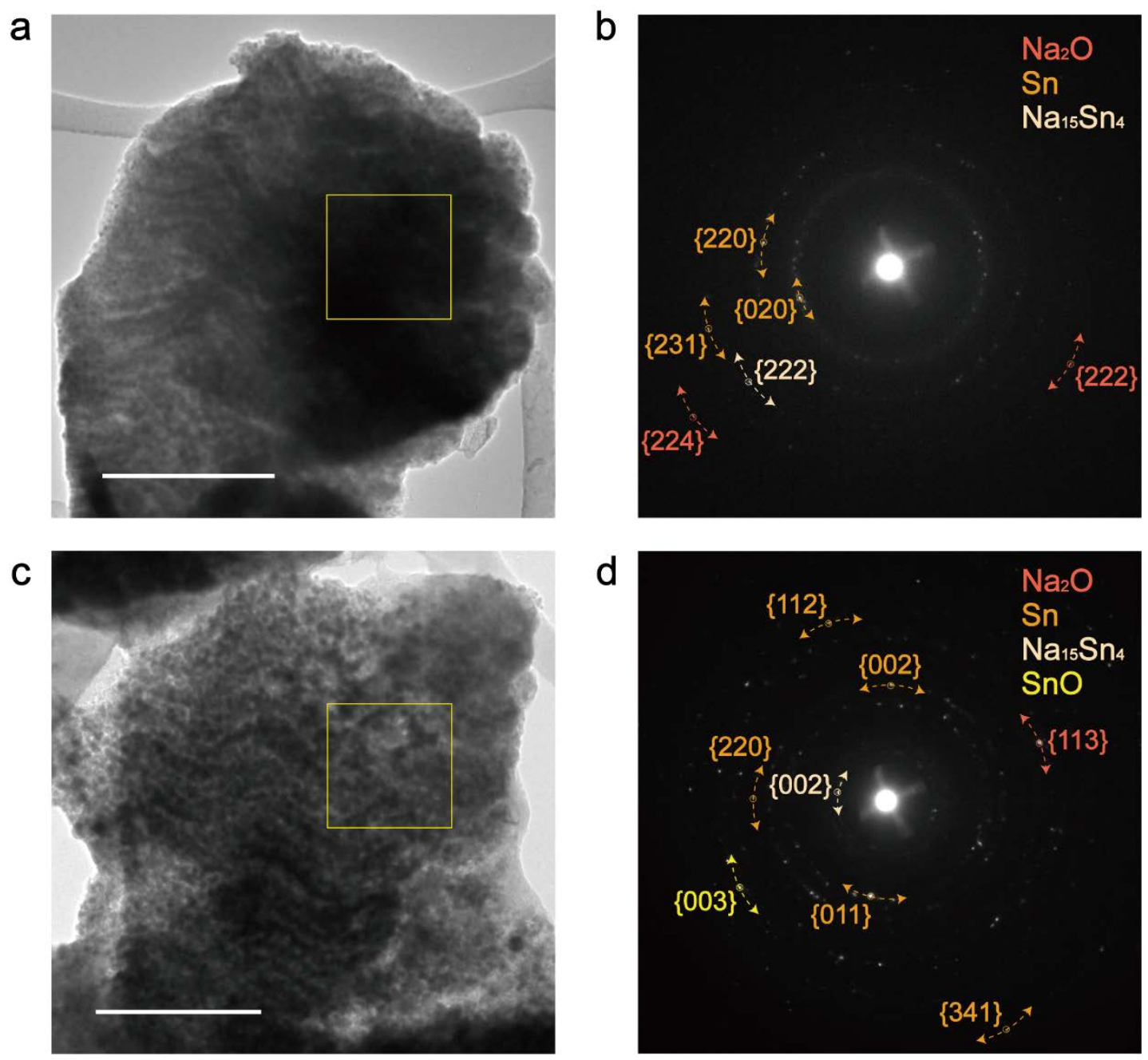

Figure S2. TEM images and corresponding SAED patterns of 3-turn a-SnO $\mathrm{NH}_{\mathrm{x}}$ electrodes at different states (a), (b) when discharged to $0.35 \mathrm{~V}$ and (c), (d) when charged to $2.5 \mathrm{~V}$. (scale bar: $0.5 \mu \mathrm{m})$ 

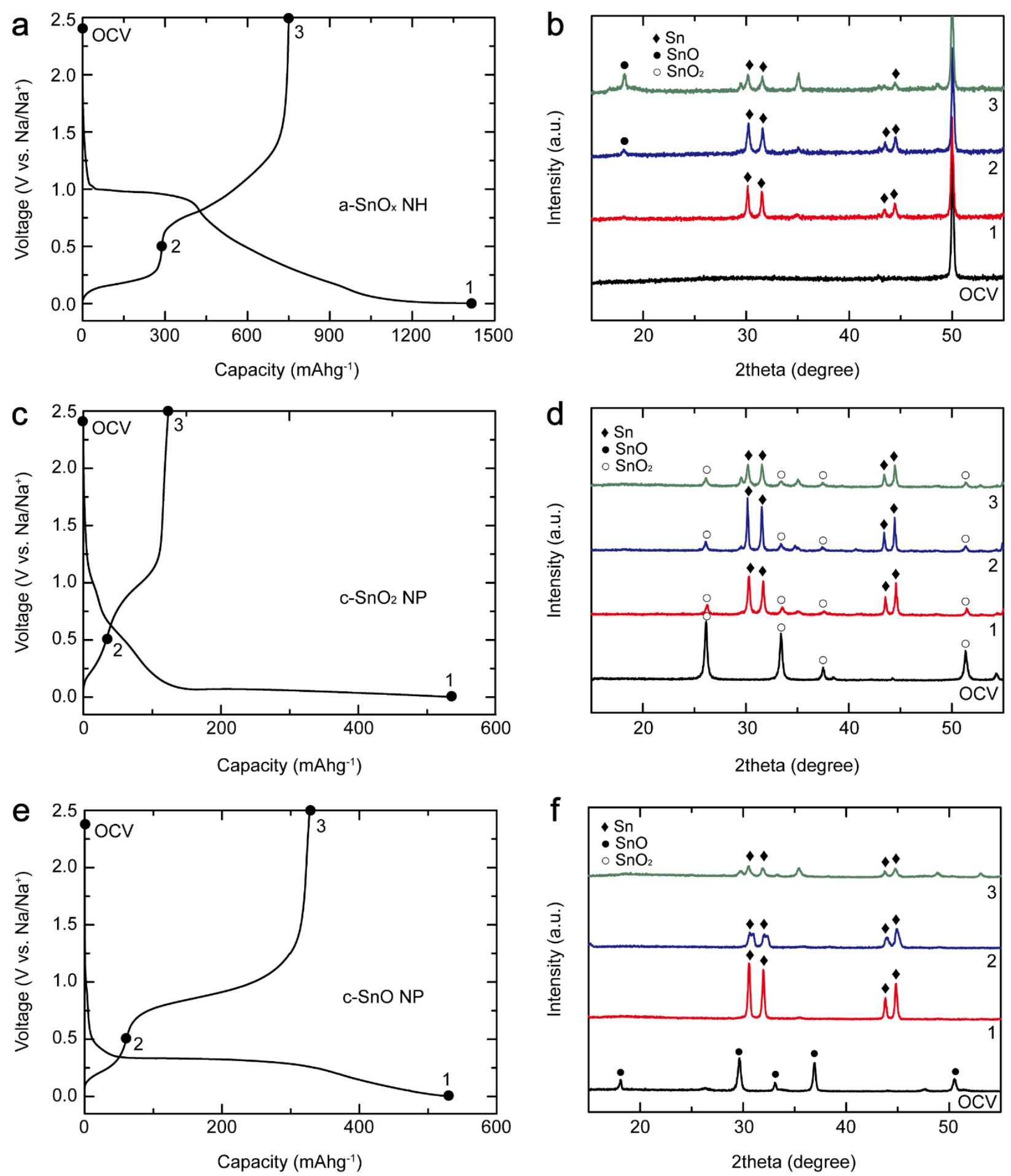

Figure S3. Discharge-charge curves for the first cycle: (a) a-SnO $\mathrm{x}_{\mathrm{NH}}$, (c) c-SnO $2 \mathrm{NP}$ and (e) cSnO NP electrodes. XRD patterns of (b) a-SnO $\mathrm{NH}$, (d) c-SnO 2 NP and (f) c-SnO NP electrodes at different states. 

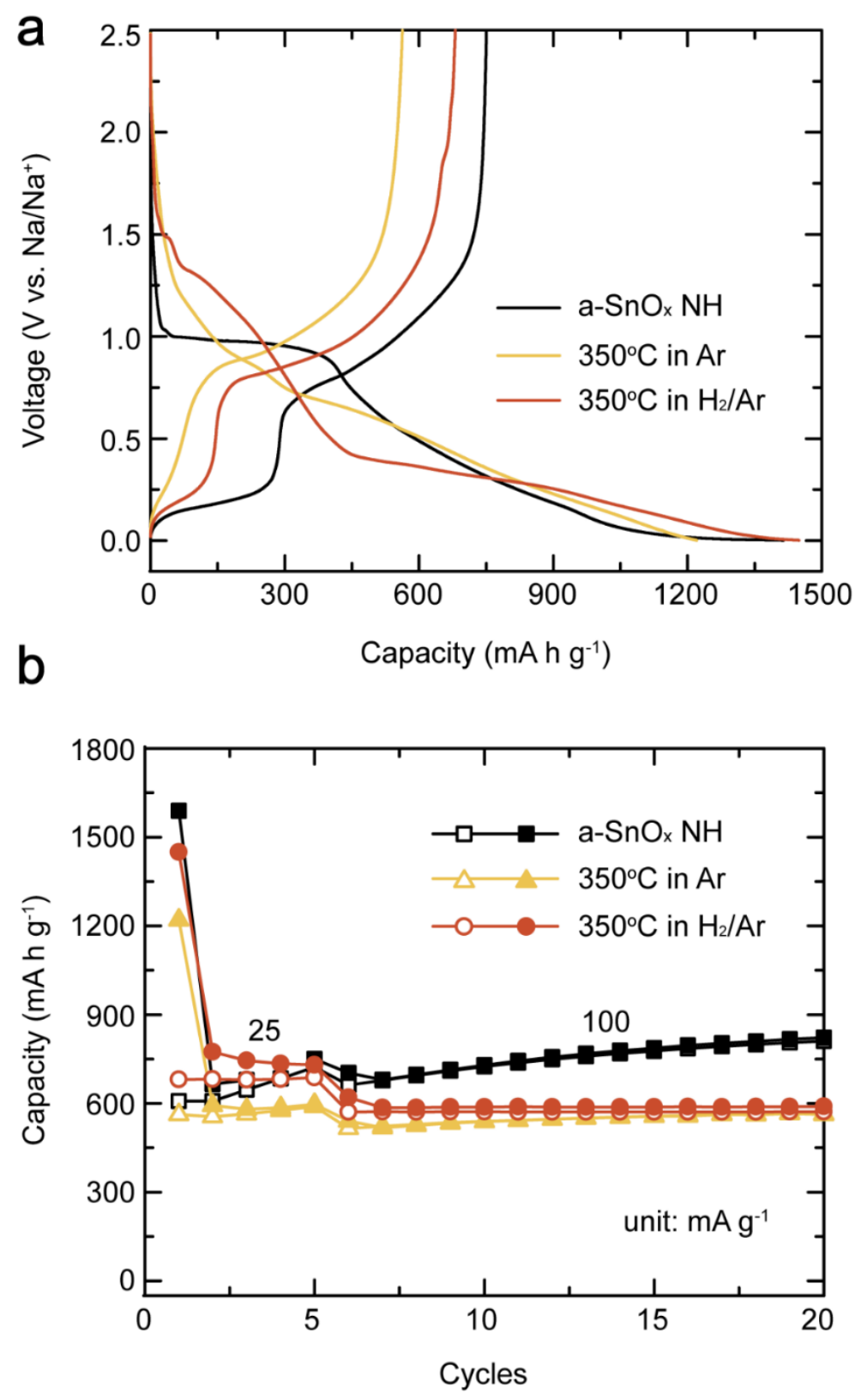

Figure S4. (a) Discharge-charge curves and (b) cycle performance (25 mA g ${ }^{-1}$ current density for the first 5 cycles and $100 \mathrm{~mA} \mathrm{~g}^{-1}$ for further cycles) of SIBs with the a-SnOx $\mathrm{NH}$ electrode and a$\mathrm{SnO}_{\mathrm{x}} \mathrm{NH}$ electrode annealed under $\mathrm{Ar}$ ambient and in $\mathrm{H}_{2} / \mathrm{Ar}(4 \%)$ ambient at $350{ }^{\circ} \mathrm{C}$ for $2 \mathrm{~h}$, respectively. 


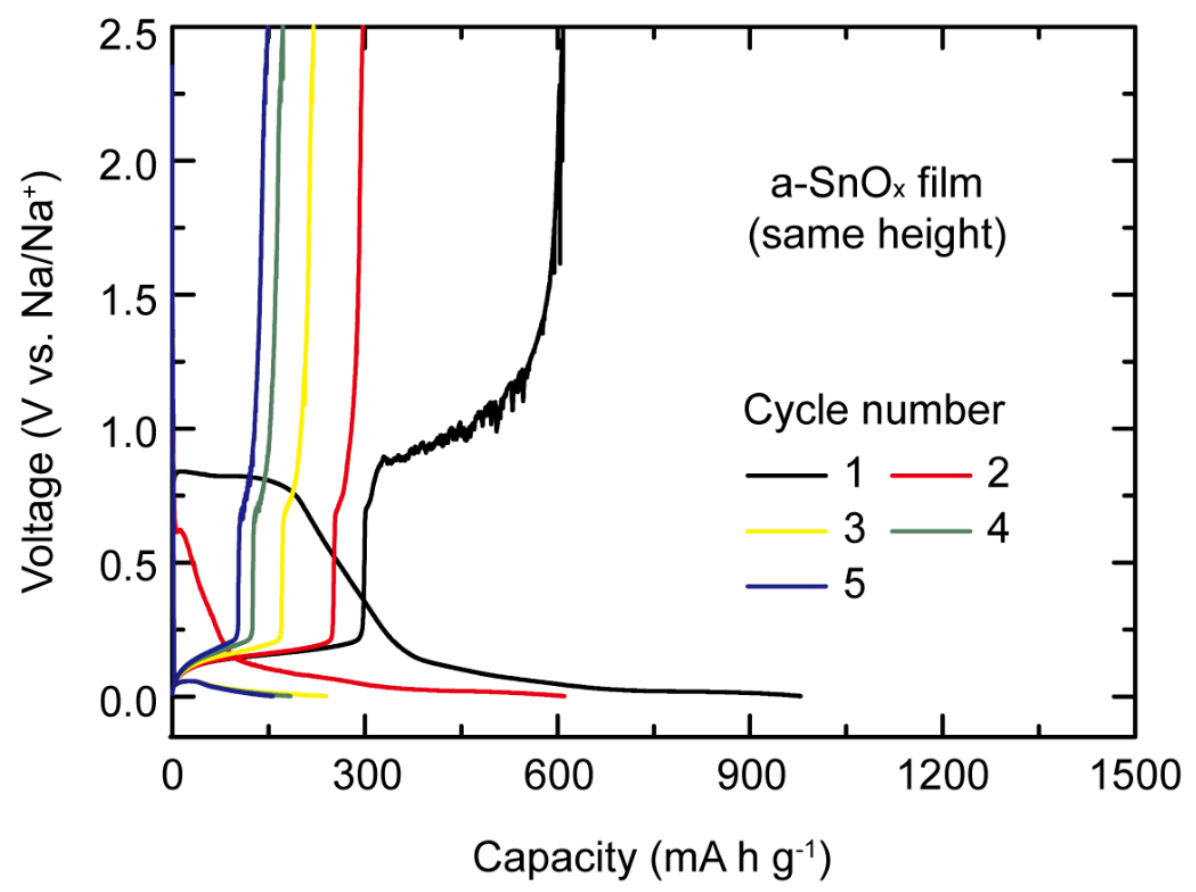

Figure S5. Discharge-charge curves of the $\mathrm{SIB}$ with a-SnOx thin-film electrode, i.e., nonnanostructured electrode, with the same height as the a-SnOx $\mathrm{NHs}$. 